Thorax 1989;44:591-593

Short report

\title{
Relapse of Pneumocystis carinii pneumonia in the upper lobes during aerosol pentamidine prophylaxis
}

\author{
R MARK BRADBURNE, DAVID B ETTENSOHN, STEVEN M OPAL, \\ F DENNIS MCCOOL
}

From the Department of Medicine, Divisions of Pulmonary Medicine and Infectious Disease, Memorial Hospital of Rhode Island, Pawtucket, and the Brown University Program in Medicine, Providence, Rhode Island, USA

\begin{abstract}
Pneumocystis carinii pneumonia was diagnosed by bronchoalveolar lavage of the upper lobes in a patient with the acquired immunodeficiency syndrome (AIDS) receiving aerosol pentamidine prophylaxis. Serendipitous availability of a normal premorbid lung gallium scan indicated that pneumocystosis had developed during aerosol pentamidine prophylaxis; at the time of presentation a repeat gallium scan indicated disease limited to the upper lobes. The relation of this unusual form of isolated upper lobe Pneumocystis carinii pneumonia to aerosol pentamidine prophylaxis warrants further investigation.
\end{abstract}

Preliminary reports support the effectiveness of aerosol pentamidine for primary prophylaxis and for prevention of relapse in Pneumocystis carinii pneumonia. ${ }^{2}$ There may, however, be some limitations to its use. Recently, a different pattern of infection, confined to the upper lung fields, has been reported. ${ }^{34}$ Chest radiography (used to specify sites of disease activity in these reports) lacks sensitivity for detection of pneumocystis pneumonia and thus does not exclude diffuse, more typical disease. ${ }^{56}$ In contrast, the gallium scan is a very sensitive indicator of pneumocystis pneumonia. ${ }^{7} \mathrm{We}$ document the development of pneumocystis pneumonia, limited to the upper lobes on gallium scanning, in a patient receiving aerosol pentamidine prophylaxis.

\section{Case report}

A 36 year old white homosexual man with the acquired immunodeficiency syndrome (AIDS) was admitted to hospital for evaluation of persistent cough and shortness of breath. One year before admission he began treatment with

Address for reprint requests: Dr David B Ettensohn, Division of Pulmonary Medicine, Memorial Hospital of Rhode Island, Pawtucket, Rhode Island 02860, USA.

Accepted 4 April 1989 zidovudine $1200 \mathrm{mg}$ per day, but soon after its initiation pneumocystis pneumonia was diagnosed by bronchoalveolar lavage. Initial treatment with intravenous trimethoprimsulfamethoxazole was stopped because of side effects (hyponatraemia and diffuse erythematous skin eruption) and intravenous pentamidine was substituted. He developed pentamidine induced pancreatitis but recovered fully and continued to take zidovudine.

Seven months before admission aerosol prophylaxis (Respirgard II nebuliser, mass median aerodynamic diameter 0.8 , geometric standard deviation $1.8 \mu \mathrm{m}$ ) was initiated with four weekly treatments (administered in the seated position) of $60 \mathrm{mg}$ followed by $60 \mathrm{mg}$ every two weeks. Four months before his main admission he was admitted to hospital because of sinusitis and treated with 14 days of intravenous cefuroxime. Resumption of aerosol pentamidine prophylaxis was planned but treatment was postponed owing to readmission one week later, when he presented with recurrent fever, myalgia, anterior thigh pain, and headache. Gallium scanning showed increased uptake in the right anterior thigh region, and the lung fields were normal (figure, A). Pyomyositis due to Staphylococcus aureus was diagnosed by needle aspiration. He recovered completely after surgical drainage and three weeks of intravenous vancomycin treatment.

One week after discharge he returned to the hospital complaining of severe abdominal pain. Pancreatitis (aetiology uncertain) was diagnosed. Resumption of aerosol pentamidine prophylaxis was again postponed.

One month before admission he had returned to his previous level of activity and resumed aerosol pentamidine prophylaxis but at a higher dose (150 mg once a month). After two weeks he developed shortness of breath, cough, and fever. Arterial blood gas analysis while he was breathing

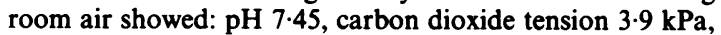
and oxygen tension $11 \cdot 1 \mathrm{kPa}$; a chest radiograph was normal. Repeat gallium scanning to evaluate the site of previous abscess formation showed no uptake in the thigh. There was, however, increased uptake in both upper lung zones (figure, B). Bronchoalveolar lavage fluid from these areas showed $P$ carinii; sputum and lavage fluid cultures showed no growth of other pathogens. Treatment with aerosol pentamidine (300 $\mathrm{mg} /$ day) alone was started because of the previous 


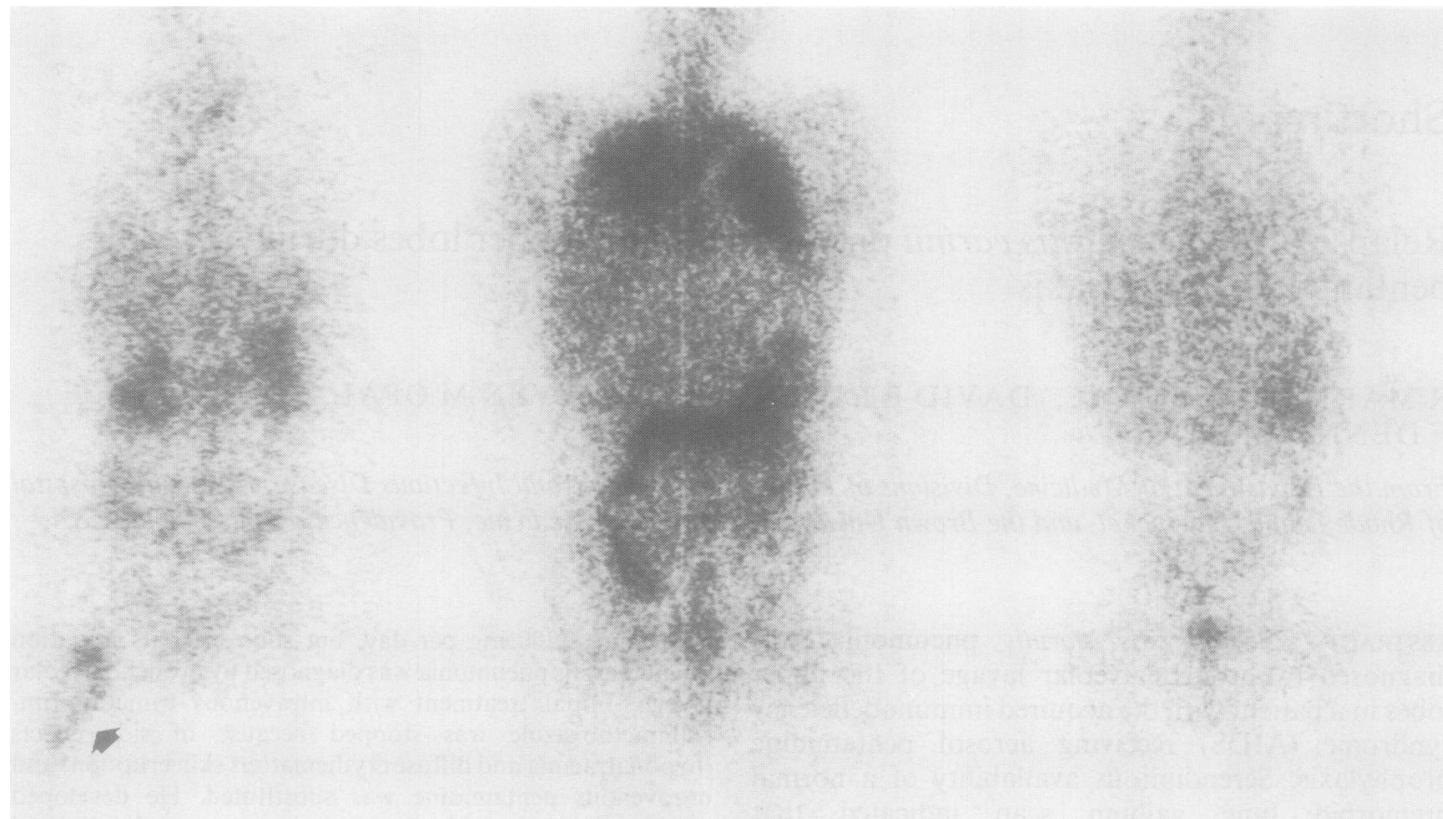

Gallium scan. A) three months prior to admission showing increased uptake in the right thigh caused by Staphylococcus aureus pyomyositis (arrow) and normal lung fields, B) during admission showing uptake in both upper lung zones due to Pneumocystis carinii pneumonia, C) two weeks after completion of therapy for Pneumocystis carinii pneumonia showing normal uptake.

adverse reactions to parenteral pentamidine and trimethoprimsulfamethoxazole. After one week his symptoms worsened and the chest radiograph showed bilateral upper lobe infiltrates. Dapsone ( $100 \mathrm{mg} /$ day) and trimethoprim ( 20 $\mathrm{mg} / \mathrm{kg} / \mathrm{day}$ ) were added. He recovered completely after two weeks. The follow up gallium scan and chest radiograph two weeks after discharge were normal (figure, $\mathrm{C}$ ).

\section{Discussion}

In a preliminary report presented at the Fourth International Conference on AIDS 24 of 255 patients were said to have relapsed with pneumocystis pneumonia while receiving aerosol pentamidine prophylaxis; in half of these the site of pulmonary infection was confined to the upper lung fields as determined by chest radiography. ${ }^{4}$ Ten per cent of patients with AIDS, however, may have normal chest radiographs in the presence of diffuse pneumocystis infection. ${ }^{6}$ Infection that is apparently limited to the upper lung fields on the chest radiograph does not therefore exclude more diffuse and thus more typical pneumocystis pneumonia.

Gallium scanning is more sensitive than chest radiography for detecting infection due to $P$ carinii pneumonia in patients with AIDS. ${ }^{67}$ In our patient, despite a normal chest radiograph, the serendipitous availability of a previous gallium scan showed that the lung abnormality confined to the upper lung zones was new. Gallium scan directed bronchoalveolar lavage showed $\boldsymbol{P}$ carinii only. After treatment the gallium scan returned to normal. The use of gallium scanning in our case documents the development of $\supset$ pneumocystis pneumonia limited to the upper lobes in a patient receiving aerosol pentamidine prophylaxis.

The mechanism leading to isolated upper lobe infection remains unknown. Abd and colleagues suggested that nonuniform aerosol deposition between the upper and the lower: lung zones may play a part. ${ }^{3}$ If so, efforts to enhance uniform deposition of aerosol by using nebulisers that produce particles of optimal size and supine positioning of patients $O$ during inhalation may be important. ${ }^{3}$ Alternatively, failure of 2 aerosol pentamidine may be related to increased pulmonary윽 clearance of the drug, as has been recently found in patients $\triangle$ with acute pneumocystis pneumonia. ${ }^{8-10}$ In our case interrup-음 tion of treatment because of intercurrent non-pneumocystis illness represents a potentially more important cause of aerosol pentamidine failure.

\section{References}

1 Feigal DW, Kandal K, Fallat R. Pentamidine aerosol prophylaxis Pneumocystis carinii pneumonia (PCP): efficacy in 211 AIDS $\stackrel{\infty}{+}$ and ARC patients. In: Abstracts of the 1988 programs and abstracts of the Twenty-eighth Interscience Conference on Antimicrobial Agents and Chemotherapy, Los Angeles. 1113.

2 Bernard EM, Schmitt HJ, Lifton A, Seltzer M, Dickmeyer MS, Armstrong D. Prevention of Pneumocystis carinii pneumonia $\mathbb{D}$ with aerosol pentamidine. In: IVth International Conference on AIDS, Stockholm. Book 1. 1988:7169.

3 Abd AG, Nierman DM, Ilowite JS, Pierson RN Jr, Loomis AL. 
Bilateral upper lobe Pneumocystis carinii pneumonia in a patient receiving inhaled pentamidine prophylaxis. Chest 1988;94:329-31.

4 Fallat R, Lowery S, Fiegal DW, Montgomery AB, Berge J. Changing patterns of Pneumocystis carinii pneumonia (PCP) on pentamidine aerosol prophylaxis (pap). In: IVth International Conference on AIDS, Stockholm. Book 1. 1988:7167.

5 DeLorenzo LJ, Huang CT, Maguire GP, Stone DJ. Roentgenographic patterns of Pneumocystis carinii pneumonia in 104 patients with AIDS. Chest 1987;91:323-7.

6 Hopewell PC, Luce JM. Pulmonary involvement in the acquired immunodeficiency syndrome. Chest 1985;137:477-8.

7 Coleman DL, Hattner RS, Luce JM, Dodek PM, Golden JA, Murray JF. Correlation between gallium lung scans and fibreoptic bronchoscopy in patients with suspected Pneumocystis carinii pneumonia and the Acquired Immune Deficiency Syndrome. Am Rev Respir Dis 1984;130:1166-9.

8 Jones DK, Higenbottam TW. Pneumocystis pneumonia increases the clearance rate of inhaled $99 \mathrm{mTc}$ DTPA from lung to blood. Chest 1985;88:631-2.

9 O'Doherty MJ, Page C, Bradbeer CS, et al. Lung 99mTc DTPA transfer and its response to treatment of PCP. In: IVth International Conference on AIDS, Stockholm. Book 1. 1988: 7158.

10 O'Doherty MJ, Page C, Bradbeer CS, et al. Lung 99mTC transfer: a criterion for selecting patients for bronchoscopy. In: IVth International Conference on AIDS, Stockholm. Book 1. 1988: 7145. 